\title{
ORIGINAL
}

\section{SEROPREVALENCIA DE INFECCIÓN POR VIRUS C DE LA HEPATITIS EN POBLACIÓN RECLUSA DEL NOROESTE DE ESPAÑA A SU INGRESO EN PRISIÓN}

\author{
Grupo Noroeste para el estudio de la Hepatitis por Virus C en el medio penitenciario. \\ Coordinador General: Vicente Martín Sánchex (1). \\ Coordinadores: Ventura Ferrer Castro (2) y José Ramón Pallas Álvarez (3). \\ Colaboradores: Luis Enrique Alonso Herrero (1); Mercedes Andrés Honorato (2); María José Coterillo \\ González(4); Luis S. García Marcos (2); José González Márquez(5); Isabel Hernández Alonso (6); Miguel \\ LLanos Gallegos (5); Eloina Mallada García (4); Mario Luis Martínez Martínez (7); Marina Morillo \\ Pérez (6); Ignacio Pérez Martínez (2); Julia Valles Martínez (7).
}

(1) Centro Penitenciario de León. (2) Centro Penitenciario de Pereiro Aguiar (Orense). (3) Centro Penitenciario de Santander. (4) Centro Penitenciario de La Coruña. (5) Centro Penitenciario de Villabona. (6) Centro Penitenciario de Villanubla. (7) Centro Penitenciario de Monterroso.

\section{RESUMEN}

Fundamento: Las prisiones españolas albergan un elevado número de personas con prácticas de riesgo para la infección por virus de la hepatitis $\mathrm{C}$ (VHC). El objeto de este trabajo es conocer la prevalencia de esta infección en la pohlación reclusa del noroeste de España y sus factores asociados.

Métodos: Se estudia a las personas ingresadas en siete centros penitenciarios del noroeste español. Se recogieron variables socio-demográficas, penitenciarias y factores de riesgo para la infección por VHC. Se realizó determinación de anticuerpos frente a VHC (EIA e INNO-LIA HCV III), virus de la hepatitis B (VHB) (EIA) y virus de la inmunodeficiencia humana (VIH) (EIA y Western-Blott). El análisis de los factores asociados se basó en regresión logística.

Resultados: El 47,9\% de los estudiados presentaron anticuerpos frente a VHC. Destacó la mayor prevalencia en: UDVP $(89.6 \%$ ). los que compartieron jeringuillas $(94 \%)$. los infectados por VIH $(92.7 \%)$, los portadores de antígeno Australia $(65,1 \%)$ y de anticuerpos frente al antígeno del core del VHB $(79.8 \%)$, aquellos con ingresos previos en prisión $(60,9 \%)$, los solteros $(54,8 \%)$, los gitanos $(52 \%)$, los trabajadores no cualificados $(50,4 \%)$, los que no habían obtenido el graduado escolar $(50,9 \%$, los tatuados (66.7\%) y aquellos con antecedentes de autolesiones $(79,3 \%)$. En el análisis de regresión logística las variables asociadas a la infección por VHC fueron: UDVP $(\mathrm{OR}=33,3 ; \mathrm{I}$.C. $95 \%=25-50)$, HBcAc (+) $(\mathrm{OR}=4.1$ : I.C. $95 \%=2.9-6.3)$, no graduado escolar $(\mathrm{OR}=1,6$ : I.C. $95 \%=1.1-2.5)$, infección por $\mathrm{VIH}(\mathrm{OR}=2,4,1 . \mathrm{C} 95 \%=1,1-5,3)$, edad (OR=0.98: I.C.95\%=0.96-1.00), meses en prisión $(\mathrm{OR}=$ $=1.011: 1 . C .95 \%=1,004-1,019)$ y antecedentes de estancias previas en prisión ( $\mathrm{OR}=2,3: \mathrm{I}, \mathrm{C} .95 \%=1,5-3,6)$.

Conclusiones: La prevalencia de infección por VHC en presos es muy elevada. Se aconseja potenciar la medidas preventivas (programas de reducción del daño) y la elaboración de protocolos de seguimiento clínico-analítico de los infectados y de tratamiento en los casos de hepatitis crónica activa para controlar este grave problema de salud pública.

Palabras clave: Hepatitis C. Epidemiología. Prisión. Salud pública. Usuario Drogas Vía Parenteral.

Correspondencia:

Vicente Martín Sánchez..

Juan de Malinas 2. 4. ${ }^{\circ} \mathrm{B}$

24005 León.

Teléfono: $987-263875$.

FAX: 987 - 202459

E-MAIL: vmartin@telprof.eurociber.es.

\section{ABSTRACT \\ Seroprevalence of Infection by Hepatitis C Virus on Entry to Prison in the Prison Population in the North-East of Spain}

Background: Spanish prisons have a high number of inmates whose behaviour puts them at risk of being infected by hepatitis $\mathrm{C}$ virus (HCV). The object of this study was to establish the prevalence of this infection and its associated factors in the prison population of the north-east of Spain.

Methods: Inmates in seven prisons in the north-east of Spain were studied. Socio-demographic and prison variables were gathered, as well as risk factors for infection by $\mathrm{HCV}$. Antibodies against HCV were determined (EIA and INNO-LIA HCV III). Hepatitis $B$ virus (VHB) (EIA), and human immunodeficiency virus (HIV) (EIA and Western-Blott). The analysis of associated factors was based on logistic regression.

Results: Of the total number of inmates studied. $47.9 \%$ presented HCV antibodies. There was greater prevalence in the case of the following: LDVP $(89.6 \%)$; those who shared needles (94\%): those infected by HIV (92.7\%); carriers of Australia antigen $(65.1 \%)$ and antibodies to the HBV core antigen $(79.8 \%)$; those who had been in prison before $(60.9 \%)$; unmarried men $(54.8 \%)$; gypsies $(52 \%)$; unqualified workers $(50.4 \%)$; those who had no basic school qualifications $(50.9 \%)$ : those with tattoos $(66.7 \%)$; and those with a background of self-inflicted injuries $(79.3 \%$ ). In the logistic regression analysis the variables associated to infection by $\mathrm{HCV}$ were: UDVP $(\mathrm{OR}=33.3 ;$ I.C. $95 \%=25-50), \mathrm{HBCAC}(+)(\mathrm{OR}=4.1: \mathrm{I} \cdot \mathrm{C} .95 \%=$ $=1.1-5.3)$, age $(\mathrm{OR}=0.98$ : I.C. $95 \%=0.96-1 .(\%)$. months in prison $(\mathrm{OR}=1.011 ; 1 . \mathrm{C} .95 \%=1.004-1.019)$ and a background of previous prison sentences $(0 R=2.3$; I.C. $95 \%=1.5-3.6)$.

Conclusions: The prevalence of infection by $\mathrm{HCV}$ in prison inmates is very high. It is therefore recommended that preventive measures be increased (harm reduction programmes) and that clinical and analysis protocols be drawn up for those infected and for treatment in cases of chronic active hepatitis in order to control this serious public health problem.

Key words: Hepatitis C. Epidemiology. Prison. Public health. Intravenous drug users. 


\section{INTRODUCCIÓN}

El virus de la hepatitis C (VHC) es la causa más frecuente de hepatitis postransfusional $^{1.2}$ y se asocia con el desarrollo de enfermedad hepática crónica, cirrosis y carcinoma hepatocelular ${ }^{3.4}$. Se encuentra muy extendido entre los usuarios de drogas por vía parenteral (UDVP) ${ }^{5}$ y dada, fundamentalmente, la gran proporción de UDVP que componen la población reclusa española, la prevalencia de infección por VHC en la misma se estima muy elevada. Por esta razón, la hepatitis por VHC constituye un grave problema de salud en las instituciones penitenciarias (IIPP) de nuestro país, y sus importantes repercusiones clínicas en la actualidad y en un futuro cercano, obligan a prestarle una especial atención.

Un grupo de facultativos de Sanidad Penitenciaria se ha constituido como grupo de trabajo, denominado Grupo Noroeste, al objeto de abordar de una manera racional y de calidad la infección por VHC en el medio penitenciario. El primer objetivo de este grupo de trabajo fue conocer la seroprevalencia de VHC en la población reclusa del noroeste español y las variables asociadas siendo este el objeto del presente trabajo.

\section{PACIENTES, MAंTERIAL Y MÉTODOS}

La población estudiada está constituida por todas las personas que ingresaron en prisión procedentes de libertad, entre el 1 de diciembre de 1994 y el 31 de mayo de 1995 y que permanecieron siete o más días en los centros penitenciarios de La Coruña, León, Monterroso (Lugo), Orense, Santander, Valladolid y Villabona (Asturias).

Se cumplimentó, mediante entrevista realizada por personal sanitario previamente adiestrado, un cuestionario validado en un pre-estudio anterior, en el que se recogían variables socio-demográficas (edad, sexo, estado civil, nivel educativo, cualificación laboral), penitenciarias (número de ingresos en prisión, edad del primer ingreso, meses de permanencia en prisión, número de autole- siones), factores de riesgo para la infección por VHC (uso de drogas por vía parenteral, patrón de uso, tatuajes) y antecedentes personales de haber padecido clínica de hepatitis.

La determinación de anticuerpos frente a los diversos virus estudiados (VHC, Virus de Hepatitis B - VHB - , y Virus de Inmunodeficiencia Humana - VIH-) se realizó según técnicas de enzimoinmunoanálisis (EIA), y confirmación en los casos positivos para las infecciones por VHC (INNO-LIA HCV III) y VIH (Western-Blott).

Los datos recogidos en el cuestionario fueron introducidos en una base de datos creada con el subprograma ENTER del programa informático EPIINFO versión 5, y los resultados se trataron estadísticamente con el subprograma ANALISYS del mismo ${ }^{6}$. El análisis estadístico se realizó con la prueba ji-cuadrado con la corrección de Yates para las variables cualitativas. Para el análisis de variables cuantitativas se utilizó el análisis de la varianza en el caso de normalidad demostrada con el test de Bartlett; en el caso de no existir homogeneidad en las varianzas, se usó la prueba no paramétrica de KuskalWallis. Para el análisis de regresión logística se utilizó el programa EGRET y el método de máxima verosimilitud ${ }^{7}$, siendo incluidas en el modelo todas aquellas variables asociadas a la infección por VHC con una $\mathrm{p}<0,01$ en el análisis univariado.

\section{RESULTADOS}

El número total de sujetos susceptibles de estudio fueron 1744. Pudo conocerse su estado respecto a la infección por $\mathrm{VHC}$ en $1214(69,6 \%)$. No pudo realizarse la extracción por libertad del interno en 290 casos $(16,6 \%)$, y se negaron a que se les realizara la extracción 240 internos $(13,7 \%)$.

El número de infectados por VHC fue de 582 personas, lo que supuso una prevalencia del $47,9 \%$ (I.C. $95 \%=45,1 \%-50,8 \%$ ).

La infección fue más frecuente en los hombres $(502 / 1030 ; 48.7 \%)$ que en las mu- 
jeres $(80 / 184 ; 43,5 \%)$, si bien las diferencias observadas no se mostraron significativas. La infección fue más frecuente en los gitanos $(52 \%)$ que en los blancos $(48 \%)$ y en los pertenecientes a otras razas o etnias $(14,2 \% ; \mathrm{p}=0,005)$; y en los solteros $(54,8 \%)$ que en los casados $(38.6 \%)$ o divorciados/separados $(40,5 \%$; $p<0,001)$. En los reclusos que tenían el Graduado Escolar la prevalencia fue inferior que en los que no lo tenían $(43,5 \%$ vs $50,9 \% ; \mathrm{p}=0,01$ ). De la misma forma, las personas cualificadas laboralmente presentaron una prevalencia inferior que las no cualificadas $(42,7 \%$ vs $50,4 \%$; $\mathrm{p}=0,01)$. (tabla 1).

Tabla 1

Distribución de la infección por VHC según diversas variables

\begin{tabular}{|c|c|c|c|c|c|}
\hline Variables & Número & $V H C(+)$ & $\%$ & $O . R$ & I.C. $95 \%$ \\
\hline \multicolumn{6}{|l|}{ Etnia } \\
\hline Blanco & $1.06^{7}$ & 513 & 48 & 5.56 & $1,7-29,6$ \\
\hline Gitano & 125 & 65 & 52 & 6.5 & $1.8-35.8$ \\
\hline Otros & 21 & 3 & 14,2 & 1 & \\
\hline \multicolumn{6}{|c|}{ Estado Civil } \\
\hline Soltero & 655 & 359 & 54,8 & 1.92 & $1,5-2.5$ \\
\hline Casado & 406 & 157 & 38,6 & 1 & \\
\hline Otrus & 143 & 58 & 40,5 & 1.08 & $0.7-1,6$ \\
\hline \multicolumn{6}{|c|}{ Graduado Escolar } \\
\hline Sí & 450 & 196 & 43,5 & 1 & \\
\hline No & 750 & 392 & 50,9 & 1,4 & $1,1-1,8$ \\
\hline \multicolumn{6}{|c|}{ Cualificación laboral } \\
\hline Sí & 388 & 166 & 42.7 & 1 & \\
\hline No & 816 & 412 & 50,4 & 1,4 & $1,1-1,8$ \\
\hline \multicolumn{6}{|l|}{ UDVP } \\
\hline Sí & 557 & 499 & 89,6 & 60,8 & $41.7-88.7$ \\
\hline No & 653 & 81 & 12,4 & 1 & \\
\hline \multicolumn{6}{|c|}{ Compartir jeringas } \\
\hline Sí & 344 & 328 & 94 & 3.4 & $1.8-6.3$ \\
\hline No & 202 & 166 & 82,2 & 1 & \\
\hline \multicolumn{6}{|l|}{ Tatuajes } \\
\hline Sí & $55^{2}$ & 369 & 66.7 & 4,2 & $3.3-5,5$ \\
\hline No & $65^{-}$ & 211 & 32.1 & 1 & \\
\hline \multicolumn{6}{|c|}{ Autolesiones } \\
\hline Si & $18:$ & 146 & 79,3 & 5.2 & $3.5-7,8$ \\
\hline No & $1.01 \mathrm{k}$ & 4.31 & 42,3 & 1 & \\
\hline \multicolumn{6}{|c|}{ Infeccion VIH } \\
\hline Sí & 19.4 & 180 & 92,7 & 20,3 & $11.2-37.2$ \\
\hline $\mathrm{No}$ & $1.00^{-}$ & 391 & 38,8 & 1 & \\
\hline \multicolumn{6}{|l|}{ Hbc Ac $1+3$} \\
\hline $\mathrm{S}_{1}$ & $47^{\circ}$ & 381 & 79.8 & 10.9 & $8.2-1+.6$ \\
\hline No & $71:$ & 190 & 26.6 & 1 & \\
\hline \multicolumn{6}{|l|}{ Primario } \\
\hline $\mathrm{Si}$ & $4(0)$ & 88 & 21.7 & 1 & \\
\hline No & 780 & 481 & 60.9 & 5.2 & $4.2-7.1$ \\
\hline
\end{tabular}


La prevalencia de infección por VHC fue significativamente más alta en los UDVP $(89,6 \%)$ que en los no UDVP $(12,4 \%)$ ( $\mathrm{p}<$ $0,001)$, y entre aquellos fue mayor en los que manifestaron haber compartido jeringuillas que entre los que lo negaron $(94 \%$ vs $82,2 \% ; \mathrm{p}<0,001)$. También fue significativamente superior en los que presentaban tatuajes $(66,7 \%$ vs $32,1 \%$; $<<0,001)$ y en los que manifestaron antecedentes de autolesiones $(79,3 \%$ vs $42,3 \%$; p < 0,001) (tabla 1).

En los infectados por VIH la prevalencia de infección por $\mathrm{VHC}$ fue superior que en los no infectados $(92,7 \%$ vs $38,8 \%$; p $<0,001)$, al igual que en los portadores del antígeno de superficie de la hepatitis B ( $\mathrm{HbsAg})(65,1 \%$ vs 47,$6 ; p=0,02$ ) y en los que presentaron anticuerpos anti-core de la hepatitis B (HbcAc) $(79,8 \%$ vs $26,6 \%$; p < 0,001). También fue superior la prevalencia en los que manifestaron antecedentes clínicos de hepatitis $(79,9 \%$ vs $38,8 \%$; $<<0,001$ ) (tabla 1 ).

La media de edad de los infectados fue significativamente inferior que en los no infectados $(27,8 \pm 6,4$ años vs $31,5 \pm 10,9$ años; $p$ $<0,001)$. En aquellos en los que este ingreso en prisión fue el primero (primario), la preva- lencia de infección por VHC fue inferior $(21,7 \%)$ que en los que tenían antecedentes previos de estancia en prisión (reincidente) $(60,9 \%)(\mathrm{p}<0,001)$ (tabla 1$)$. Los reclusos infectados por VHC presentaban una mayor precocidad al ingreso en prisión $(21,7 \pm 5,4$ años vs $28,8 \pm 11,3$ años; $\mathrm{p}<0,001$ ), un mayor número de ingresos $(4,9 \pm 4,6$ vs $2,2 \pm 2,6$; $\mathrm{p}<0,001)$ y mayor tiempo de permanencia en la misma $(24,5 \pm 39$ meses vs $7,5 \pm 20$ meses; $p<$ 0.001 ) (tabla 2).

No se encontraron diferencias significativas en la prevalencia de la infección por VHC entre los distintos centros penitenciarios que participaron en el estudio (rango $36,5-53,3 \%, p=0,364$ ) (tabla 3 ).

En el modelo de regresión logística las variables asociadas a la infección por el VHC fueron: nivel socio-cultural, anticuerpo del core de VHB ( $\mathrm{HbcAc}$ ), infección por $\mathrm{VIH}$, ser o no interno primario, ser UDVP, la edad y los meses de permanencia en prisión. Presentaron infección por VHC más frecuentemente aquellos sujetos que no habían obtenido el graduado escolar, los UDVP, los que tenían antecedentes de estancias previas en prisión, los que habían permanecido en ella más tiempo, los infectados

Tabla 2

Análisis de variables cuantitativas asociadas a la infección por VHC

\begin{tabular}{|c|c|c|c|c|c|}
\hline Variables & Media & $D E$ & Mediana & $P 25 \cdot P 75$ & $P$ \\
\hline \multicolumn{6}{|l|}{ Edad } \\
\hline VHC $(+)$ & 27,8 & 6,4 & 27 & $24-31$ & \multirow{2}{*}{$<0,001$} \\
\hline VHC (-) & 31,5 & 10,9 & 29 & $23-28$ & \\
\hline \multicolumn{6}{|c|}{ Edad $1^{\mathrm{er}}$ ingreso } \\
\hline VHC (+) & 21,7 & 5,4 & 20 & $17-25$ & \multirow[t]{2}{*}{$<0,001$} \\
\hline VHC (-) & 28,8 & 11,3 & 26 & $20-34$ & \\
\hline \multicolumn{6}{|c|}{$N .^{0}$ de ingresos } \\
\hline $\mathrm{VHC}(+)$ & 4,9 & 4,6 & 4 & $2-6$ & \multirow[t]{2}{*}{$<0,001$} \\
\hline VHC (-) & 2.2 & 2,6 & 1 & $1-2$ & \\
\hline \multicolumn{6}{|c|}{ Meses en Prisión } \\
\hline $\mathrm{VHC}(+)$ & 24,5 & 39,1 & 7 & $1-30$ & \multirow[t]{2}{*}{$<0,001$} \\
\hline VHC (-) & 7,5 & 20,1 & 1 & $1-3$ & \\
\hline
\end{tabular}


Tabla 3

Prevalencia de la infección por VHC en los distintos centros penitenciarios

\begin{tabular}{|lcccc|}
\hline Centro & Número & VHC $(+)$ & $\%$ & I.C. $95 \%$ \\
\hline La Coruña & 179 & 81 & 45,3 & $38,0-52,5$ \\
León & 111 & 47 & 42,3 & $33,2-51,5$ \\
Monterroso & 52 & 19 & 36,5 & $23,6-51,0$ \\
Orense & 90 & 48 & 53,3 & $42,5-63,9$ \\
Santander & 192 & 95 & 49,5 & $42,4-56,6$ \\
Villanubla & 226 & 114 & 50,4 & $43,9-57,0$ \\
Villabona & 364 & 178 & 48,9 & $43,8-54,0$ \\
\hline
\end{tabular}

por VIH y los que presentaron anticuerpos anti-HBc . Por el contrario los más viejos presentaron infección por VHC con menor frecuencia (tabla 4).

\section{DISCUSIÓN}

La prevalencia de infección por VHC observada es muy superior a la hallada en otros estudios hechos en población donan$\mathrm{te}^{4.8-13}$, más de 20 veces superior a la estimada para la población general de nuestro país $(0,1-2 \%)^{14-16}$ y similar e incluso ligeramente inferior, en algunos casos, a la observada por otros autores en población reclusa ${ }^{17-21}$. La elevada proporción de UDVP (el $46 \%$ de los sujetos incluidos en el estudio declararon ser o haber sido UDVP) y ser esta variable la que presenta una mayor asociación con la infección podría explicar en gran medida lo elevado de la prevalencia de infección por VHC observada en estas prisiones.
Los niveles de infección por VHC observados en el colectivo de UDVP fueron superiores a los obscrvados en la mayoría de los estudios publicados, si bien las prevalencias oscilaban entre el $37,5 \%$ y el $93,8 \%{ }^{14.15,22-36}$.

La elevada prevalencia encontrada en los no UDVP podría explicarse al menos parcialmente, en la ocultación del consumo de drogas por parte de algunos internos y por que el porcentaje de UDVP fuera incluso superior al declarado. Aún en este caso, la prevalencia encontrada en los no UDVP siguc sicndo muy elevada y obliga a pensar en otros posibles mecanismos de transmisión. La transmisión sexual del VHC parece ser posible, pero menos efectiva que para VIH o VHB como se recoge en varios estudios $^{8,14,25,31,34,37,38}$; puede abundar en ello el no haber observado diferencias en la distribución de la infección por VHC entre hombres y mujeres; diferencias que en este colectivo si se han observado en la distribución de la

Tabla 4

Factores asociados a la infección por VHC. Análisis de regresión logística

\begin{tabular}{|lccccc|}
\hline Variables & Coeficiente & $\begin{array}{c}\text { Error } \\
\text { estandar }\end{array}$ & Odds Ratio & I.C. 95\% & Valor de P \\
\hline No Graduado escolar & 0,49 & 0,49 & 1,64 & $1,09-2,47$ & 0,019 \\
HbcAc (+) & 1,42 & 0,21 & 4,17 & $2,94-6,25$ & $<0,001$ \\
VIH (+) & 0,86 & 0,4 & 2,38 & $1,08-5,26$ & 0,033 \\
UDVP Sí & 3,51 & 0,21 & 33,3 & $25,0-50,0$ & $<0,001$ \\
Edad & $-0,02$ & 0,01 & 0,98 & $0,96-1,0$ & 0,036 \\
Meses en prisión & 0,01 & 0,004 & 1,01 & $1,004-1,019$ & 0,003 \\
Primario No & 0,84 & 0,22 & 2,32 & $1,46-3,60$ & $<0,001$ \\
\hline
\end{tabular}


infección por VIH $^{39}$ y se han achacado a la transmisión heterosexual, más frecuente en las mujeres, de la infección VIH. No se ha entrado a valorar los hábitos sexuales de la población objeto de estudio, pero la promiscuidad sexual y el escaso uso de medidas de protección en las relaciones sexuales nada infrecuentes en este colectivo pudieran contribuir a la elevada prevalencia de infección encontrada.

En la población gitana se ha observado una mayor prevalencia de infección que puede obedecer a múltiples razones: una posible transmisión horizontal de la infección en condiciones de pobreza y hacinamiento extremas tal y como se ha descrito para la infección por $\mathrm{VHB}^{40}$; más frecuencia en los comportamientos de riesgo o prácticas más arriesgadas; o que provengan de un medio de más elevada prevalencia donde a igualdad de comportamientos de riesgo la probabilidad del contagio es mayor como se ha explicado la mayor infección por VIH detectada también en este colectivo ${ }^{41}$.

La asociación de la infección por VHC con la infección por VHB y por VIH ya ha sido descrita por otros autores tanto en población general o infectada por VIH y/o VHB $^{10,15.31,34,42.43}$ como en UDVP ${ }^{10.22 .24 .27 .32 .44}$ y en población reclusa ${ }^{20}$. En todos los casos las prevalencias de co-infección encontrada son similares a las descritas en este trabajo, siendo preocupantes la prevalencia de coinfección VHC-VIH por sus importantes repercusiones clínicas. El que las tres infecciones víricas compartan mecanismos de transmisión y lo extendida de estas infecciones en estos colectivos puede explicar la asociación encontrada.

La mayor prevalencia de infección por VHC en aquellos sujetos que no han obtenido el Graduado Escolar pudiera ser debido a que éstos presentaran un mayor grado de marginalidad y una menor capacidad de percepción del riesgo, lo que daría lugar a prácticas de riesgo más frecuentes y exten- didas. Este hallazgo esta en consonancia con lo informado por otros autores ${ }^{13,45}$.

Con respecto a la edad, parece encontrarse en población general un incremento de prevalencia hasta los 40 años, descendiendo por encima de esa edad, lo que podría explicarse por una menor exposición al virus en edades avanzadas, mortalidad previa por enfermedad hepática de los infectados y/o por la desaparición de niveles detectables de anticuerpos $^{46}$. La media de edad de la población que ha participado en nuestro estudio es inferior a 40 años, y son los más jóvenes los que presentan una mayor prevalencia de infección, lo que puede ser debido a la juventud del colectivo más afectado, los UDVP.

La asociación del tiempo de estancia en prisión con la infección por VHC ha sido informada ya por algunos autores en nuestro país ${ }^{19}$. Un mayor tiempo de permanencia en prisión supone una mayor probabilidad de consumir drogas, la falta de material estéril de inyección obliga a compartirlo y el control del personal de vigilancia sobre una práctica prohibida en los centros penitenciarios predispone al consumo en condiciones antihigiénicas; todo ello unido a la elevada prevalencia de la infección en el medio penitenciario, supone una situación de muy alto riesgo para contraer la infección.

La mayor prevalencia de la infección en personas que han ingresado varias veces en prisión puede traducir características diferenciales de los UDVP reincidentes frente a los reclusos primarios y/o situaciones de consumo de alto riesgo derivado de los síndromes de abstinencia sufridos al ingreso en prisión.

Como conclusión, constatamos la elevada prevalencia de infección por VHC en la población reclusa estudiada. Si tenemos presente que más del $50 \%$ de los pacientes con hepatitis $C$ evolucionara hacia la cronicidad. de los que un 20-30\% presentarán una cirrosis $^{47}$, y un porcentaje considerable de ellos desarrollarán un carcinoma hepatocelular primario, nos encontramos ante un grave 
problema de salud en las instituciones penitenciarias que puede justificar el potenciar las medidas encaminadas a prevenir la infección, tales como los programas de reducción de riesgos dirigidos a los UDVP, la búsqueda activa de infectados y el seguimiento clínico y analítico de los anti-VHC positivos para establecer un diagnóstico precoz de los casos de hepatitis crónica que permita el tratamiento con interferón.

\section{AGRADECIMIENTOS}

\begin{abstract}
Al Dr. J.A. Caylá, del Instituto Municipal de la Salud de Barcelona, por su colaboración en la realización e interpretación del análisis multivariante.
\end{abstract}

\section{BIBLIOGRAFÍA}

1. Alter HJ, Purcell RH, Holland PV, Feinstone SM, Morrow AG, Moritsugu Y. Clinical and serological analysis of transfusion-associated hepatitis. Lancet 1990; 2: 838-841.

2. Dienstag JL. Non-A, non-B hepatitis. Recognition, epidemiology and clinical features. Gastroenterol $1983 ; 85: 439-462$.

3. Esteban JI, López-Talavera JC, Genesca J, Madoz P, Viladomiu L, Muñiz E, et al. High rate of infectivity and liver disease in blood donors with antibodies to hepatitis $\mathrm{C}$ virus. Ann Intern Med $1991 ; 115: 443-449$.

4. Llibre JM, Bartolí M, Berrocal M, González-Moreno $M$. Seroprevalencia del virus de la hepatitis $\mathrm{C}$ en adictos a drogas por vía parenteral. Med Clín (Barc) 1992; 99: 675.

5. Dean AD, Dean JA, Burton AH, Dicker RC. Epi Info, versión 5: A word processing, database, and statistics program of epidemiology on microcomputers. Stone Mountain, Georgia: USD Incorporated; 1990.

6. Epidemiological Graphics, Estimation and Testing Package (EGRET). Seattle, Washington: Statistics and Epidemiology Research Corporation; 1990.

7. Widell A, Sundstrom G, Hansson BG, Moestrup N, Nordenfelt E. Antibody to hepatitis-C-virusrclated proteins in sera from alanine-aminotransferase-screened blood donors and prospectively studicd recipients. Vox Sang 1991; 60: 28-33.
8. Martín AM, Canut A, Corral M, Cordero M. Seroprevalencia del virus de la hepatitis $\mathrm{C}$ en donantes y grupos de alto y bajo riesgo de Salamanca. Med Clín (Barc) 1992; 98: 676-677.

9. Suárez A, San Román F, Rodríguez M, Rodrigo L. Modificaciones en la prevalencia del anti-VHC en donantes de sangre en Asturias. Med Clin (Barc) 1992; 99: 515 .

10. Iraporda V, Gil L, Moreda J, Fernández P, González J, Velicia R. Donantes de sangre con antiHCV positivo. Factores de riesgo. Rev Esp Enferm Dig 1995; 87: 179-180.

11. Murphy EL, Bryzman S, Willians AE, Co-Chien $\mathrm{H}$, Schreiber GB, Ownby HE, Gilcher RO, Kleinman SH, Matijas L, Thomson RA, Nemo GJ. Demographic determinants of hepatitis $C$ virus seroprevalence among blood donors. JAMA 1996; 275: $995-1000$.

12. Van der Poel CL, Reesink HW, Mauser-Bunschoten Ep, Kaufmann RH, Leentvar-Kuypers A, Chamuleau RA, et al. Prevalence of anti-HCV antibodies confirmed by recombinant aimmunoblot in diferent population subsest in The Netherlands. Vox Sang 1991, 61 (1): 30-6.

13. Hess G, Rossol S, Voth R, Schutt H, Meyer zum Busdenfelde KH. Diagnose der Hepatitis-C-Virus (HCV)-Infektion: Diagnostische Wertigkeit des Anti-HCV-Test. Z Gastroenterol May 1990; 28 (5): $251-2$.

14. Bruguera M. Hepatitis vírica aguda. Medicine, 6 (8): $311-322$.

15. Ranieri R, Passaretti B, Capoccia A, Vccchi C, Molella AM. Prevalenza del virus dell'epatite C in un gruppo di detenuti sieropositivi per HIV. Col Mal Infect Parassit 1991, 43 (2): 137-41.

16. Pallas JR, Ruiz JC. Infecciones víricas trasmitidas por vía parenteral. Prevalencia en el C.P. de Santander. Libro de ponencias y comunicaciones I Congreso Nacional de Sanidad Penitenciaria. León 18, 19 y 29 Nov; 1993. p. 259.

17. Añón C, Del Olmo JA, Llovet F, Molina J, Ballester A. Prevalencia de hepatitis $C$ en el Centro Penitenciario de preventivos de Valencia. Libro de ponencias y comunicaciones I Congreso Nacional de Sanidad Penitenciaria. León 18, 19 y 20 Nov; 1993. p.320.

18. García-Villanueva M, Conde A, Gainza MJ, Sola $\mathrm{P}$, Chamarro A. Infección por VIH y por los virus de la hepaitits B y C en interno de un Centro Penitenciario. Libro de ponencias y comunicaciones I Congreso Nacional de Sanidad Penitenciaria. León 18, 19 y 20 Nov. 1993. p. 320. 
19. Crofts N, Stewart T, Hearne P, Ping XY, Breshkin AM, Locarnini SA. Spread of bloodborne viruses among Australian prison entrants. BMJ 1995; 310: 285-288.

20. Gloria H, Ramalho F, Marinho R, et al. Infeccoes viricas em toxicodependentes de drogas endovenosas. Significado clinico e prognostico. Acta Med Port 1991; 4(5):263-7.

21. Trubner K, Polywka S, Puschel K; Lanfs R. Hepatitis $\mathrm{C}$ in deceased drug addicts. Int $\mathrm{J}$ Legal Med 1991, 104 (5): 251-4.

22. Zeldis JB, Jain S, Kuramoto IK, et al. Seroepidemiology of viral infections among intravenous drug users in northen California. West J Med Jan 1992; $156(1): 30-5$.

23. Donahue JG, Nelson KE, Munoz A, et al. Antibody to Hepatitis $\mathrm{C}$ virus among cardiac surgery patients, homosexual men, and intravenous drug users in Baltimore, Maryland. Am J Epidemiol 1991, 134 (10): 1206-11.

24. Polywka S, Lanfs R. Hepatitis $C$ antibodics among different group at risk and patient with suspected non-A, non-B hepatitis. Infection 1991; 19(2): 81-4.

25. Pont J, Neuwald C, Salzner G. Antibody prevalence of parenterally transmitted viruses HIV-1, HTLV-1, HBV, HCV in Austrian intravenous frug users. Infection 1991; 19(6): 427-30.

26. Verdier D, Boissonnas A, Lebon P, Christoforov B, Cremer GA. Marqueurs de l'hepatite B et C chez le toxicomane IV: relation avec la presence D'anticorps antio. VIH et le taux de gammaglobulines seriques. Rev Med Interne (France) 1991:12/6 Suppl.

27. Neuwald C, Pont J, Tomasit J, Baner K. Antikorperpravalenz fur Hepatitis $C$ und weiterer parenteral ubertragbarer viruserkranhugen bei i.v. Drogennabhangigen. Acta Med Austriaca 1992; 19 (2): $47-8$.

28. Anand C, Fonseca $\mathrm{K}$, Walle RP, Powell S, Willians M. Antibody to hepatitis $C$ virus in selected groups of a Canadian urban populatio. Int J Epi demiol 1992; 21 (1): 143-5.

29. García-Samaniego J, Enríquez A, Soriano V, Muñoz F. Prevalencia de anticuerpos frente al virus de la hepatitis $\mathrm{C}$ en sujetos VIH positivos de diferentes grupos de riesgo. Med Clin (Barc) 1992; 99: 357-58.

30. Llibre JM, Bartrolí M, Berrocal M, GonzálezMoreno M. Seroprevalencia del virus de la hepatitis $\mathrm{C}$ en adictos a drogas por vía parenteral. Med Clin (Barc) 1992; 99: 675.
31. García-Boyer FA, Enríquez O, Sauri V, Beneto JL, Lozano F. Hepatitis crónicas en ADVP: Seroprevalencia de Ac VHC y coinfección con VIH y marcadores de hepatitis B y D. Libro de ponencias y comunicaciones I Congreso Nacional de Sanidad Penitenciaria. León 18, 19 y 20 Nov. 1993. p.320.

32. Tor J, Llibre JM, Carbonell M, Muga R, Ribera, Soriano V, et al. Sexual transmission of hepatitis $C$ virus and its relation with hepatitis $B$ virus and HIV see commentes. BMJ Nov 17 1990, $301(6761): 11303$.

33. Fingerhood MI, Jasinski DR, Sullivan JT. Prevalence of hepatitis $\mathrm{C}$ in a chemically dependent population. Arch Intern Med 1993; 153: 2025 2030.

34. Utsumi T, Hashimoto E, Okumura Y, Takayanagi M, Nishikawa H, Kigawa M, Kumakuma N, Toyo kawa $\mathrm{H}$. Heterosexual activity as a risk factor for the transmission of hepatitis $\mathrm{C}$ virus. $J$ Med Virol 1995; 46: 122-125.

35. Gasparinni V, Chiaramonte M, Moschen ME, Fabris $\mathrm{P}$, Altinier $\mathrm{G}$, Majori $\mathrm{S}$, et al. Hepatitis $\mathrm{C}$ virus infection in homosexual men: a seroepidemiological study in gay clubs in north-east Italy. Eur $\mathbf{J}$ Epidemiol Nov. 1991; 7 (6): 665-9.

36. Tedder RS, Gilson RJ, Briggs M, Loveday C, Cameron $\mathrm{CH}$, Garson JA, et al. Hepatitis $\mathrm{C}$ virus: evidence for sexual transmission. BMJ Jun 1 1991; 302 (6788): 1299-302.

37. Martín V, Caylà $\mathrm{J} \Lambda$, Morís ML, Alonso LE, Pérez R. Evolución de la prevalencia de infección por Virus de la Inmunodeficiencia Humana en población reclusa al ingreso en prisión. Años 19911995. Rev Esp Salud Publica 1997; 269-280.

38. Bayas JM, Bruguera M, Martín V, . Ilepatitis B y delta en reclusos jóvenes. Med Clin (Barc) 1990; 94:164-168.

39. Martín V, Bayas JM, Laliga A, Pumarola T, Vidal J, Jiménez de Anta MT, et al. Seropedidemiology of HIV-1 infection in a Catalonian penitentiary. AIDS 1990; 4:1023-1026.

40. Portillo JD, García Martín P, Alvarez Rojas, et al Infección por el virus de la hepatitis B, C y delta en portadores del virus de la inmunodeficiencia humana. Med Clin. (Barc.)1991; 96: 245-247.

41. De Miguel J, Collazos J, Mayo J, Lopez MJ, Echaniz C, Mendarte U. Seroprevalencia del virus delta $y$ de la hepatitis $C$ en pacientes con infeccion cronica por el virus de la hepatitis B. Rev Clín Esp 1994; 194: 897-900.

42. Levine OS, Vlahov D, Koehler J, Cohn S, Spronk AM, Nelson KE. Seroepidemiology of hepatitis B virus in a population of injecting drug users. Asso 
ciation with drug injection patterns. Ain J Epidemiol 1995; 142: $331-341$.

43. Thomas DL, Mahley RW, Badur S, Palaoglu E, Quinn TC. The epidemiology of hepatitis $C$ in Turkey. Infection 1994; 22: 411-414.
44. Laguna del Estal P. Infección por VHC: seroepidemiología y diagnóstico serológico. An Med Intern (Madrid) $1993 ; 10(1): 38-46$.

45. Sanchez Tapias JM. Hepatitis crónica. Medicine 6 (8): 323-331. 